a diagnosis is difficult. ${ }^{21}$ Although magnetic resonance imaging can lead to important changes in management, these occur in a small minority of patients suspected of having an intracranial lesion. The benefits of magnetic resonance imaging are therefore likely to be measured ultimately more by how it fulfils its promise of replacing invasive techniques such as myelography ${ }^{22.3}$ and diagnostic cerebral angiography ${ }^{24}$ than by its performance compared with computed tomography of the head.

We thank our colleagues in the departments of neurology, neurosurgery, neuroradiology, and clinical physics for their cooperation and collaboration and for contributing to collecting the data; Drs K W Grossart and E Teasdale for reporting many of the computed tomograms; Professor A Williams and Dr J Hutton, department of health economics, University of York, for fruitful discussions and collaboration; and $\mathrm{Dr} G$ Murray, department of medical statistics, University of Glasgow, for advice on analysis. The magnetic resonance imaging unit in the Institute of Neurological Sciences, Glasgow, was established with support from the Medical Research Council, the Scottish Hospital Endowment Research Trust, the chief scientist, the Scottish Home and Health Department, the Greater Glasgow Health Board, and the Institute of Neurological Sciences Research Trust.

1 Kent DL, Larson EB. Magnetic resonance imaging of the brain and spine -is clinical efficacy established after the first decade? Ann Intern Med 1988;108: $402-24$

2 Hadley DM, Teasdale GM. Survey of progress: magnetic resonance imaging of the brain and spine. $\mathcal{F}$ Neurol 1988;235:193-206.

3 Hillman BJ. Evaluating magnetic resonance: what do we need to know? Invest Radiol 1986;21:289-91. (Critical review.)

4 Cooper LW, Thomas MPH, Chalmers C, et al. The poor quality of early evaluations of magnetic resonance imaging. $\mathcal{F} A M A$ 1988;259:3277-80.

5 Sheps SB. Technological imperatives and paradoxes. FAMA 1988;259:3312-3.
6 Kent DL, Larson EB. Diagnostic technology assessments: problems and prospects. Ann Intern Med 1988;108:759-61.

Bydder GM, Steiner RE, Thomas DJ, et al. Nuclear magnetic resonance imaging of the posterior fossa: 50 cases. Clin Radiol 1983:34:173-88.

8 Hadley DM, Macpherson P, Grant R, Paterson J, Lawrence A. Magnetic resonance imaging in the posterior fossa. A prospective analysis of 200 patients. In: Hopf MA, ed. Proceedings of the third congress of the European Society of Magnetic Resonance in Medicine and Biology. Aberdeen: European Society of Magnetic Resonance in Medicine and Biology, 1986:94.

9 Everitt BS. The analysis of contingency tables. London: Chapman and Hall, 1977.

10 Smith T. Taming high technology. Br Med f 1984:289:393-4.

11 Institute of Medicine Committee for Evaluating Medical Technologies in Clinical Use. Assessing medical technologies. Washington, DC: National Academy Press, 1986

12 Jennett B. Assessment of clinical technologies: importance for provision and use. International fournal of Technology Assessment in Health Care 1988;4: $435-45$.

3 Jennett B. High technology medicine: benefits and burdens. 2nd ed. Oxford: Oxford University Press, 1986.

14 Ranshoff DF, Feinstein AR. Problems of spectrum and bias in evaluating the efficacy of diagnostic tests. $N$ Engl f Med 1978:299:926-30.

15 Brandt-Zawadzki M, Norman D, Newton H, et al. Magnetic resonance of the brain: the optimal screening technique. Radiologv 1984;152:71-7

16 Bradley WG, Waluch V, Yadley RA, Wycoff RR. Comparison of CT and MR in 400 patients with suspected disease of the brain and cervical spinal cord. Radiology 1984;152:695-702.

17 Haughton VM, Rimm AA, Sobocinski KA, et al. A blinded clinical comparison of MR imaging and CT in neuroradiology. Radiology 1986;160 $751-5$.

18 McCartney FJ. Diagnostic logic. Br.Med f 1987;295:1325-31

9 Balla JI, Elstein AS, Christensen C. Obstacles to acceptance of clinical decision analysis. Br.Med f 1989;298:579-82.

20 Bydder GM. Clinical application of gadolinium-DTPA. In: Stark DD, Bradley WG, eds. Magnetic resonance imaging. St Louis: C V Mosby, 1988:182-200.

21 Duncan R, Hadley DM, Teasdale GM. Magnetic resonance scanners. Lancet $1988 ; \mathrm{i}: 60-1$

22 Hyman RA, Gorey MT. Imaging strategies for MR of the spine. Rudiol Clin North Am 1988;26:505-33

23 Thomson JLG. Experiences at the new magnetic resonance imaging centre at Bristol. Br F Radiol 1989;62:134-7.

24 Dumoulin CL, Hart HR. Magnetic resonance angiography. Radiology 1986;161:717-20.

(Accepted 18 May 1989)

\title{
An unusual uveitis in Tanzanian children
}

\author{
David Yorston, Allen Foster, John Treharne
}

\begin{abstract}
In 1982-7, 254 children with panuveitis were seen at Mvumi Hospital, Tanzania, representing $56 \%$ of all cases of uveitis seen. Half were aged under 2. No consistent abnormality accounted for the uveitis and it resolved spontaneously over 6-12 weeks. A trial of prednisolone was performed in 30 children: 18 showed improvement by four weeks compared with 20 of 35 controls given only topical steroids and mydriatics.
\end{abstract}

\section{Mvumi Hospital, PO Dodoma, Tanzania \\ David Yorston, FRCS, ophthalmologist}

\section{Institute of \\ Ophthalmology, London ECIV 9EJ \\ Allen Foster, FRCS, senior lecturer in preventive ophthalmology \\ Institute of \\ Ophthalmology, London WC1 \\ John Treharne, MRCPATH, reader in virology}

Correspondence to: $\mathrm{Dr}$ Foster.

BrMed f 1989;299:355-7

\section{Introduction}

Uveitis in children is fairly unusual, representing $1-20 \%$ of all cases of uveitis. ${ }^{1-3}$ Uveitis may be divided anatomically into anterior, peripheral (pars planitis), and posterior. In analysing 150 cases in children Perkins found a ratio of anterior to peripheral to posterior uveitis of about $3: 2: 1$, with the main causes of posterior uveitis or panuveitis being toxoplasmosis $(38 \%)$ and toxocariasis (11-20\%); the cause of $24 \%$ of cases was not known.

\section{Patients and methods}

From 1982 to 1987,254 children aged 0-9 years with posterior uveitis or panuveitis were seen at Mvumi Hospital in central Tanzania. All were seen in the outpatient clinic and were admitted and examined by AF or DY. Examination included slit lamp microscopy (in older children) and direct and indirect ophthalmoscopy (sometimes performed under sedation or general anaesthesia) in all children. A tap of the anterior chamber was performed in six patients undergoing examination under anaesthesia. The aqueous humour obtained was examined for the presence and type of cells. Where indicated, investigations included a thick blood film (for malaria and tick borne relapsing fever), peripheral blood count, and examination of urine and stools by microscopy.

Serum samples from 10 children with uveitis and nine controls matched for age and sex were examined at the Institute of Ophthalmology, London. Antibodies to herpes simplex virus and Epstein-Barr virus were screened with an indirect immunofluorescence test using the virus grown in cell culture as antigen. Antibodies to HIV were screened by enzyme linked immunosorbent assay (ELISA) (Welcozyme, Wellcome), and serology for toxoplasmosis was carried out by a latex screen test.

\section{Results}

Of patients with uveitis seen from 1982 to 1987,254 were aged under $10(128(50 \%)$ aged under 2 years and $33(13 \%)$ aged 6 months or under) and 201 were aged 10 or over $(67$ aged $10-19,59$ aged $20-29,45$ aged $30-39$, 17 aged $40-49$, and 13 aged 50 or over). Of the children aged under 10 years, 124 were boys and 130 were girls.

The clinical presentation was an acute red eye or a grey-white opacity in the pupil that had been noticed by a parent, or both. The children were generally well. Ocular examination showed an acute anterior uveitis with cells, keratic precipitates, and occasionally hypo- 
pyon. The iris seemed normal and there were no lens opacities. A characteristic finding was extremely dense vitreous infiltrates, which usually totally obscured the red reflex. The findings were bilateral in 28 of $78(36 \%)$ children aged under 1 year and 35 of $176(20 \%)$ aged 1-9 years. Several infants with bilateral dense vitreous infiltrates presented with nystagmus.

The clinical course of panuveitis is of spontaneous resolution over six to 12 weeks. Detailed ocular examination during resolution showed vitreous "snowballs" and pars plana "snowbanks" in the pars plana but no focal chorioretinal lesions. Examination after resolution showed normal retinas in most patients with no focal lesions to suggest toxoplasmosis or toxocariasis. Premacular fibrosis was seen in a few patients.

Peripheral blood counts and thick blood films showed no consistent abnormality, although a history of fever was given for some of the patients. Urine and stool microscopy showed infections with the common parasitic worms (ascaris and hookworm) and occasionally Giardia lamblia but no consistent abnormality to explain the uveitis in most children. There was no evidence of tuberculosis - many children had received BCG immunisation shortly after birth. Aqueous humour from six patients showed a mixture of lymphocytes and polymorphic cells, but further immunological studies on the aqueous humour were not possible at the time.

The results of serology from 10 patients and 9 controls matched for age and sex showed no significant difference between cases and controls in the numbers of patients positive for toxoplasmosis, herpes simplex virus, or Epstein-Barr virus. All patients were negative for HIV antibody and rheumatoid factor.

A randomised trial of systemic steroids was carried out in 30 children with 35 children acting as controls. The controls received topical steroids and mydriatics, while the cases received topical steroids, mydriatics, and oral prednisolone $1 \mathrm{mg} / \mathrm{kg} /$ day for one week, after which the dose was halved over four weeks so that by the fifth week treatment was stopped. Eighteen of the 30 children given systemic steroids $(60 \%)$ showed definite improvement at the end of four weeks, compared with 20 of the 35 who were not given systemic steroids $(57 \%)$.

\section{Discussion}

Previous studies have reported uveitis in children (usually defined as aged $0-15$ years) as accounting for $1 \cdot 3-19 \cdot 4 \%$ of all cases of uveitis, with an average of about $6 \%$ in most series. ${ }^{1.3}$ Of all cases, $7-96 \%$ have been reported to be anterior uveitis.' In Perkins's series $51 \%$ of 150 children had posterior or generalised panuveitis.' Our series is atypical in that uveitis in children aged 0-9 represents $56 \%$ of all cases seen, and half of these occur in the first two years of life.

The condition is seen throughout the year, with $40 \%$ of cases occurring in the four months from June to September. There may be a geographical distribution, with many similar cases in Iringa, Shinyanga, and Dareda but few in Mbeya and Sumbawanga (figure; J Taylor and $M$ Scudder, personal communications). Similar cases have been seen in Zambia (J Cairns, personal communication).

Mvumi Hospital is in a rural village of about 10000 people, of whom about 3000 are aged under 10 years. In 1985,1986 , and $1987,17,14$, and 18 children with panuveitis from Mvumi village were seen, which represents an incidence of $5 \cdot 4 / 1000$ children/year. If these figures remain constant each child in Mvumi has a chance of developing uveitis in the first 10 years of life of about 1 in 20

Toxoplasmosis is the most common presumptive diagnosis in childhood posterior uveitis, being responsible for $28-62 \%$ of cases. ${ }^{2}$ Although patients with the typical features of toxoplasmosis have been seen at Mvumi Hospital, the clinical appearances in these children, particularly the absence of any focal retinal lesions, would not support this diagnosis. Also, the uveitis was seen in children negative for toxoplasmosis on the latex screen test.

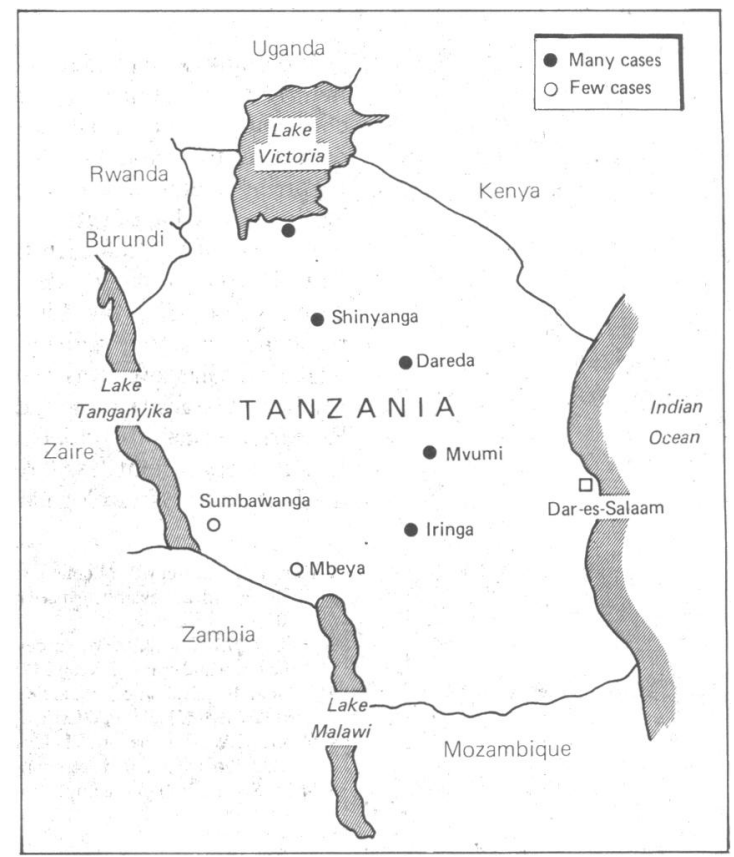

Distribution of cases of panuveitis in Tanzania

Toxocara canis can cause posterior segment inflammation and granuloma formation in young children. It is usually unilateral. Perkins reported that $11 \%$ of his patients had toxocariasis and a further $9 \%$ possibly were infected with toxocara. ${ }^{2}$ Serology for toxocara has not been carried out on our children, but the clinical appearances and spontaneous resolution would not support this diagnosis.

None of the children in this series had arthropathy suggestive of juvenile chronic arthritis or ankylosing spondylitis. ${ }^{4}$ Both these conditions are uncommon in east Africa. Serology for syphilis was not routinely carried out, but syphilis is not common in this rural population, and a syphilitic uveitis would be unlikely to resolve spontaneously. All 10 serum samples tested for HIV antibody were negative.

We believe this to be a new form of panuveitis in children, occurring particularly in infants and consisting of acute anterior uveitis with severe vitritis, no retinal lesions, and spontaneous resolution over six to 12 weeks. The term panuveitis seems more appropriate than peripheral uveitis or pars planitis as the children have a dense vitreous infiltrate with cellular response in the anterior chamber. This response may be "overspill," but it can be sufficiently severe to cause a hypopyon and sometimes presents as an acute red eye.

The prognosis for recovery of vision is good in most patients, the exceptions being a few children who develop premacular fibrosis and small children with absent red reflexes for two to three months who may develop amblyopia. We have not yet seen evidence of recurrent disease. Treatment with oral prednisolone seems of no benefit, although topical steroids can be used to treat anterior uveitis. Mydriatics have routinely been given to all patients.

The cause of this unusual uveitis is not known. The clinical picture does not support a diagnosis of toxoplasmosis, toxocariasis, or uveitis associated with 
arthritis. Anecdotal evidence that the children often present in clusters, together with some data in support of a geographical distribution, suggests that this uveitis may be a response to a parasitic, viral, or spirochaetal infection met early in life. A preceding fever in some children and the mixture of lymhocytes and polymorphic cells in the aqueous humour further supports this idea.

We thank Christoffel Blindenmission, West Germany,
Miss M Patel for laboratory analysis, and Miss Suzanne Chawner for secretarial help.

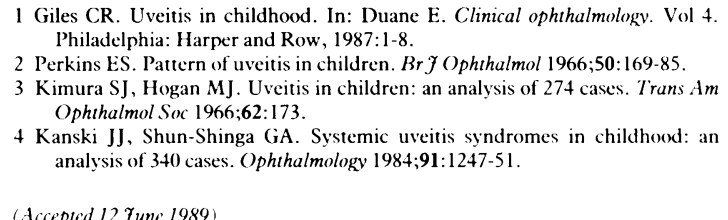

\title{
Respiratory symptoms as predictors of 27 year mortality in a representative sample of British adults
}

\author{
Lucy Carpenter, Valerie Beral, David Strachan, Kristie L Ebi-Kryston, Hazel Inskip
}

Department of

Epidemiology and

Population Sciences, London School of Hygiene and Tropical Medicine, University of London, London WC1E 7HT Lucy Carpenter, MSC, lecturer

David Strachan, MRCP, lecturer

Imperial Cancer Research Fund, Cancer

Epidemiology and Clinical Trials Unit, University of Oxford, Gibson Building, Radcliffe Infirmary, Oxford OX2 6HE

Valerie Beral, MRCP, director

Department of

Environmental and

Preventive Medicine, St

Bartholomew's Hospital,

Medical College, London EC1M 6BQ

Kristie L Ebi-Kryston, PHD, statistician

Medical Research Council Laboratories, Fajara, Near Banjul, The Gambia, West Africa

Hazel Inskip, PHD,

statistician

Correspondence to: $\mathrm{Ms}$ Carpenter.

BrMedf 1989;299:357-61

\section{Abstract}

Objective-To examine associations between reported respiratory symptoms (as elicited by questionnaire) and subsequent mortality.

Design-Prospective cohort study.

Setting-92 General practices in Great Britain.

Participants -A nationally representative sample of 1532 British men and women aged between 40 and 64.

Main outcome measures-Mortality from all causes, cardiovascular disease, lung cancer, and chronic bronchitis.

Results-Subjects were interviewed in 1958 regarding various respiratory symptoms (including cough, phlegm, breathlessness, and wheeze) by using a questionnaire which formed the basis of the Medical Research Council's questionnaire on respiratory symptoms. By the end of 1985, 889 deaths had been reported, including 51 in men due to chronic bronchitis. After adjustment for differences in age and smoking habits death rates from chronic bronchitis in men who reported symptoms were greater than those in men who did not for each of the symptoms examined. The adjusted mortality ratios were $3.4(95 \%$ confidence interval 1.8 to 6.5$)$ for morning cough, $3.7(2.0$ to 6.9$)$ for morning phlegm, $6.4(3.0$ to 13.8$)$ for breathlessness when walking on the level, and $10.5(4.4$ to 24.6$)$ for wheeze most days or nights. Mortality ratios were also significantly raised for four episodic symptoms not usually included in more recent respiratory symptom questionnaires-namely, occasional wheeze (mortality ratio $6.0 ; 95 \%$ confidence interval, 2.4 to $15 \cdot 1$ ), weather affects chest $(5 \cdot 7 ; 3 \cdot 1$ to $10 \cdot 3)$, breathing different in summer $(4.9 ; 2.8$ to 8.6$)$, and cold usually goes to chest $(3 \cdot 7 ; 2.0$ to $6 \cdot 8)$. The excess mortality associated with these symptoms remained significant after further adjustment for breathlessness or phlegm. Ratios for all cause mortality in men and women were also significantly raised for most respiratory symptoms, death rates being some 20 $50 \%$ higher in people reporting symptoms after adjustment for age, sex, and smoking. Breathlessness was the only symptom significantly associated with excess mortality from cardiovascular disease (mortality ratio $1.4(95 \%$ confidence interval 1.0 to 1.9) for breathlessness when walking on the level). Ratios were generally around unity and not significant for mortality due to lung cancer.

Conclusions-The results suggest that episodic symptoms, which often do not appear in standard respiratory questionnaires, predict subsequent mortality from chronic obstructive airways disease.
This supports the hypothesis that reversible airflow obstruction may be a precursor of progressive and irreversible decline in ventilatory function.

\section{Introduction}

In the 1950s there was concern about the high level of morbidity and mortality from chronic bronchitis in Britain; between 1957 and 1961 the death rate from bronchitis was $108 / 100000$ in men aged 45-64 in England, Wales, and Scotland, which was about 20 times the rate in Denmark and Norway. ${ }^{1}$ Within the United Kingdom pronounced variations in mortality were recorded, higher rates being found in urban areas and the lower social classes. ${ }^{23}$ One study undertaken at that time to discover the reason for these variations was a survey of respiratory symptoms in a random sample of men and women attending general medical practices in Britain. ${ }^{+}$The questionnaire developed for that survey subsequently served as the basis for the Medical Research Council's questionnaire on respiratory symptoms, which is widely used today. The people who were surveyed in 1958 by using the original questionnaire have been followed up to the end of 1985. We describe the associations between their reported respiratory symptoms and their subsequent death rates.

\section{Subjects and methods}

Details of the original survey have been reported. ${ }^{4}$ Briefly, the sample consisted of adult men and women chosen at random from the lists of general practitioners in rural and urban areas of England, Wales, and Scotland. Stratified random samples of one man and one woman in each of the five year age groups 40-44, 45-49, 50-54, 55-59, and 60-64 were drawn from each participating practice. Each practice undertook to interview at least one such sample of 10 subjects. If a sampled subject had died or moved a substitute of the same sex was selected at random from the appropriate age group. Of the 1630 people thus sampled and available for examination at the time of the survey in $1958,1569(96 \%)$ were interviewed. Data relating to 442 wives of sampled men, which were presented in the original report, are not included here because they did not form a representative sample of women in the population.

The questionnaire used in the survey subsequently served as the prototype for the Medical Research Council's questionnaire on respiratory symptoms, though it underwent some revisions in 1966, 1976, and $1986 .^{56}$ During this process some of the original 